\title{
On Rough and Smooth Neighbors
}

\author{
William D. BANKS, Florian LUCA, \\ and Igor E. SHPARLINSKI
}

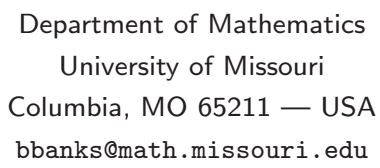

Department of Computing

Macquarie University

Sydney, NSW 2109 - Australia

igor@ics.mq.edu.au

Received: November 2, 2005

Accepted: June 19, 2006

\section{ABSTRACT}

We study the behavior of the arithmetic functions defined by

$$
\mathcal{F}(n)=\frac{P^{+}(n)}{P^{-}(n+1)} \quad \text { and } \quad \mathcal{G}(n)=\frac{P^{+}(n+1)}{P^{-}(n)} \quad(n \geq 1),
$$

where $P^{+}(k)$ and $P^{-}(k)$ denote the largest and the smallest prime factors, respectively, of the positive integer $k$.

Key words: smallest prime divisor, largest prime divisor.

2000 Mathematics Subject Classification: 11N25, 11N64.

\section{Introduction}

For every integer $n \geq 2$, let $P^{+}(n)$ and $P^{-}(n)$ denote the largest and the smallest prime factors of $n$, respectively; put $P^{+}(1)=1$ and $P^{-}(1)=\infty$. An integer $n$ is said to be $y$-smooth if $P^{+}(n) \leq y$, and it is said to be $z$-rough if $P^{-}(n)>z$.

There are several papers in the literature which study smoothness properties of consecutive integers. In certain ranges, upper and lower bounds have been obtained on the number of positive integers $n \leq x$ for which $P^{+}(n(n+1)) \leq y$, and other 
similar questions have been studied; see, for example, $[4,5,10,15]$. The arithmetic function

$$
\mathcal{H}(n)=\frac{P^{+}(n)}{P^{+}(n+1)} \quad(n \geq 1)
$$

has been investigated in $[3,6]$; in particular, it is known (see [6]) that for every $\varepsilon>0$ there exists $\delta>0$ such that the inequalities

$$
n^{-\delta} \leq \mathcal{H}(n) \leq n^{\delta}
$$

hold for at most $\varepsilon x$ positive integers $n \leq x$. The distribution of integers $n$ for which $P^{+}(n)<P^{+}(n+1)$ (that is, $\left.\mathcal{H}(n)<1\right)$ and that of integers $n$ such that $P^{+}(n)>$ $P^{+}(n+1)$ have also been studied, as well as analogous questions about the possible orderings among the three primes $P^{+}(n), P^{+}(n+1)$, and $P^{+}(n+2)$; see [3,6]. These results suggest that the values of $P^{+}(n)$ and $P^{+}(n+1)$ are essentially independent.

In this paper, we introduce and study the arithmetic functions

$$
\mathcal{F}(n)=\frac{P^{+}(n)}{P^{-}(n+1)} \quad \text { and } \quad \mathcal{G}(n)=\frac{P^{+}(n+1)}{P^{-}(n)} \quad(n \geq 1),
$$

for which we obtain a variety of results with a similar flavor; our results suggest that the values of $P^{+}(n)$ and $P^{-}(n \pm 1)$ are essentially independent; that is, the smoothness of $n$ does not affect the roughness of its neighbors $n \pm 1$.

We show that for almost all positive integers $n$, the values $\mathcal{F}(n)$ and $\mathcal{G}(n)$ are "large" in a certain sense. This is consistent with our intuition: Since the set of $y$-smooth integers $s \leq x$ is much smaller than the set of $y$-rough integers $r \leq x$ over a wide range in the $x y$-plane (see [14, chapters III.5 and III.6]), for "random" integers $s, r$ it is likely that $P^{+}(s)$ is much larger than $P^{-}(r)$. Our results show that the same result is true when $s$ and $r$ are neighbors, that is, when $|s-r|=1$.

Although $\mathcal{F}(n)$ and $\mathcal{G}(n)$ tend to be large, the value sets $\mathcal{F}(\mathbb{N})$ and $\mathcal{G}(\mathbb{N})$ are quite dense in the set of all positive real numbers. In particular, both value sets contain all fractions of the form $p / q>1$ and almost all fractions of the form $p / q<1$, where $p$ and $q$ are prime numbers. On the other hand, we show that for every prime $p$, there are infinitely many primes $q>p$ such that $p / q \notin \mathcal{F}(\mathbb{N})$, and we expect the same statement to hold for $\mathcal{G}(\mathbb{N})$ as well.

In addition to their intrinsic interest as natural analogues of the arithmetic function $\mathcal{H}(n)$, the functions $\mathcal{F}(n)$ and $\mathcal{G}(n)$ also exhibit interesting links with some famous sets of positive integers, such as the Fermat and Mersenne primes.

\section{Notation}

Throughout the paper, any implied constants in symbols ' $O$,' ' $\ll$, and ' $\gg$ ' are absolute unless specified otherwise. We recall that, for positive functions $U$ and $V$, the statements $U=O(V), U \ll V$, and $V \gg U$ are all equivalent to the assertion that $U \leq c V$ holds with some constant $c>0$. 
In what follows, the letters $\ell, p, q$ and $r$ (with or without subscripts) always denote prime numbers, $k, m$ and $n$ always denote positive integers, and $x$ is always a positive real number. As usual, we let $\pi(x)$ denote the number of primes $p \leq x$.

Finally, for any real number $x>0$ and integer $k \geq 1$, we denote by $\log _{k} x$ the $k$-th iterate of the function $\log x=\max \{\ln x, 1\}$, where $\ln x$ is the natural logarithm.

\section{Value sets}

Let $\mathcal{F}(\mathbb{N})$ and $\mathcal{G}(\mathbb{N})$ denote the collection of values taken by $\mathcal{F}(n)$ and $\mathcal{G}(n)$, respectively, as $n$ varies over the set of natural numbers $\mathbb{N}$. The following result shows that the intersection $\mathcal{F}(\mathbb{N}) \cap \mathcal{G}(\mathbb{N})$ contains every fraction of the form $p / q$, where $p, q$ are primes with $p>q$ :

Theorem 2.1. For any two primes $p>q$, there exist integers $m, n \in \mathbb{N}$, with

$$
\max \{m, n\} \leq \exp (p+o(p)) \quad \text { as } \quad p \rightarrow \infty
$$

such that

$$
\mathcal{F}(m)=\mathcal{G}(n)=p / q
$$

Proof. Let $\mathcal{L}=\{$ primes $\ell \leq p: \ell \neq q\}$, and put

$$
L=\prod_{\ell \in \mathcal{L}} \ell
$$

Let $M$ be the unique integer such that $1 \leq M<q$ and $L M \equiv 1(\bmod q)$, and put

$$
m=(q-1) L M \quad \text { and } \quad n=(q+1) L M-1 .
$$

Since $p \geq q+1>M$, it is clear that $P^{+}(m)=P^{+}(n+1)=p$. On the other hand, it is easy to see that $q \mid m+1$ and $q \mid n$, whereas

$$
m+1 \equiv 1 \quad(\bmod \ell) \quad \text { and } \quad n \equiv-1 \quad(\bmod \ell) \quad(\ell \in \mathcal{L})
$$

therefore, $P^{-}(m+1)=P^{-}(n)=q$. Combining these results, it follows that $\mathcal{F}(m)=$ $\mathcal{G}(n)=p / q$.

By the Prime Number Theorem, we also have the bound

$$
\max \{m, n\}<(q+1) L M \leq\left(q^{2}-1\right) L<q \prod_{\ell \leq p} \ell=\exp (p+o(p)),
$$

and this finishes the proof.

Remark 2.2. Using explicit bounds from [12] for the product of the primes $\ell \leq p$, one can derive from (1) an entirely explicit version of Theorem 2.1 with a specific function of $p$ in the exponent rather than $p+o(p)$. 
Remark 2.3. A minor modification to the construction of Theorem 2.1 allows one to build infinitely many $m$ and $n$ with $\mathcal{F}(m)=\mathcal{G}(n)=p / q$ when $p>q$. On the other hand, the equation $\mathcal{H}(m)=p / q$ has only finitely many solutions $m$ since by a classical result of C. Siegel [13] it is known that $P^{+}(n(n+1)) \rightarrow \infty$ as $n \rightarrow \infty$. (See also [9] for the currently best known effective lower bound of the type $P^{+}(n(n+1)) \gg$ $\log _{2} n \log _{3} n / \log _{4} n$.)

In contrast with Theorem 2.1 , the value set $\mathcal{F}(\mathbb{N})$ does not contain every fraction of the form $p / q$ with $p<q$ (see Theorem 2.5 below), and we expect the same to be true for $\mathcal{G}(\mathbb{N})$. However, the next result implies that almost all such fractions occur in the intersection $\mathcal{F}(\mathbb{N}) \cap \mathcal{G}(\mathbb{N})$.

Theorem 2.4. For every pair of primes $(p, q)$ such that $p<q \leq x$, with at most $o\left(\pi(x)^{2}\right)$ possible exceptions, there exist integers $m, n \in \mathbb{N}$, with

$$
\max \{m, n\} \leq \exp (\exp (q+o(q))) \quad \text { as } \quad q \rightarrow \infty,
$$

such that

$$
\mathcal{F}(m)=\mathcal{G}(n)=p / q
$$

Proof. Let $y=\sqrt{\log x}$. We exclude from consideration any pair of primes $(p, q)$ for which $p \leq q / y$; clearly, there are at most

$$
\pi(x) \pi(x / y) \ll \frac{x}{\log x} \frac{(x / y)}{\log (x / y)} \ll \frac{x^{2}}{(\log x)^{2.5}}=o\left(\pi(x)^{2}\right)
$$

such pairs with $p<q \leq x$. We also exclude those pairs $(p, q)$ for which

$$
\max \left\{P^{+}(q-1), P^{+}(q+1)\right\}>q / y \text {. }
$$

To estimate the number of such pairs, we apply Brun's method (see, for example, $[8$, Theorem 2.3]) to deduce that for every positive integer $a$, each of the linear forms $a \ell+1$ and $a \ell-1$ take prime values for at most

$$
N_{a}(x) \ll \frac{x}{\varphi(a) \log ^{2}(x / a)} \ll \frac{x \log _{2} a}{a \log ^{2}(x / a)}
$$

primes $\ell \leq x / a$, where $\varphi(\cdot)$ is the Euler function. In the above estimate, we have used the bound $a / \varphi(a) \ll \log _{2} a$, which holds uniformly for all $a \geq 1$. If $p<q \leq x$ and $P^{+}(q \pm 1)>q / y$, then $q=a \ell \mp 1$ for some integer $a<2 y$ and prime $\ell \leq(x+1) / a$; hence, there are at most

$$
\pi(x) \sum_{a<2 y} 2 N_{a}(x+1) \ll \pi(x) \frac{x \log y \log _{2} y}{\log ^{2} x} \ll \pi(x)^{2} \frac{\log _{2} x \log _{3} x}{\log x}=o\left(\pi(x)^{2}\right)
$$

such pairs of primes $(p, q)$. 
Now, fix one of the remaining pairs $(p, q)$. Let $\mathcal{L}=\{$ primes $\ell \leq p\}$ and $\mathcal{R}=$ \{primes $r: p<r<q\}$, and put

$$
m=(q-1) L^{(q-1) R} \quad \text { and } \quad n=(q+1) L^{(q-1) R}-1,
$$

where

$$
L=\prod_{\ell \in \mathcal{L}} \ell \quad \text { and } \quad R=\prod_{r \in \mathcal{R}}(r-1) .
$$

Since $P^{+}(q \pm 1) \leq q / y<p$, we have $P^{+}(m)=P^{+}(n+1)=p$. We claim that $P^{-}(m+1)=P^{-}(n)=q$ (and consequently, $\left.\mathcal{F}(m)=\mathcal{G}(n)=p / q\right)$. Indeed, using Fermat's Little Theorem, we have

$$
m \equiv-L^{(q-1) R} \equiv-1 \quad(\bmod q),
$$

hence, $q \mid m+1$. Similarly,

$$
n \equiv L^{(q-1) R}-1 \equiv 0 \quad(\bmod q),
$$

thus, $q \mid n$. On the other hand, as $(r-1) \mid R$ for each prime $r \in \mathcal{R}$, Fermat's Little Theorem also implies that

$$
m+1=(q-1) L^{(q-1) R}+1 \equiv q \not \equiv 0 \quad(\bmod r),
$$

and

$$
n=(q+1) L^{(q-1) R}-1 \equiv q \not \equiv 0 \quad(\bmod r),
$$

thus, $r \nmid(m+1) n$. Finally, since $\ell \mid L$ for every $\ell \in \mathcal{L}$, it is clear that $\ell \nmid(m+1) n$, and the claim is proved.

By the Prime Number Theorem, we have the estimates

$$
L \leq \exp (p+o(p)) \quad \text { and } \quad R \leq \exp (q+o(q)),
$$

and the theorem follows.

The following result shows that $\mathcal{F}(\mathbb{N})$ does not include all fractions of the form $p / q$ with $p<q$ :

Theorem 2.5. For every prime $p$, let

$$
\mathcal{Q}_{p}=\{\text { primes } q: p / q \notin \mathcal{F}(\mathbb{N})\} .
$$

Then,

$$
\#\left\{q \leq x: q \in \mathcal{Q}_{p}\right\} \gg \pi(x),
$$

where the implied constant depends only on p. Moreover,

$$
\min _{q \in \mathcal{Q}_{p}}\{q\} \leq \exp (O(p)) .
$$


Proof. For a fixed prime $p$, let $q$ be a prime such that:

(i) every prime $\ell \leq p$ is a quadratic residue modulo $q$;

(ii) -1 is a quadratic nonresidue modulo $q$.

We claim that $q \in \mathcal{Q}_{p}$. Indeed, if $n \geq 1$ is an integer for which $P^{+}(n)=p$, property (i) implies that $n$ is a quadratic residue modulo $q$. But then the equation $P^{-}(n+1)=q$ is not possible, for otherwise $n \equiv-1(\bmod q)$ is a quadratic nonresidue by (ii).

To construct examples of such primes $q$, let $N=4 \prod_{\ell \leq p} \ell$, and let $a$ be the congruence class modulo $N$ determined by the conditions $a \equiv 7(\bmod 8)$ and $a \equiv$ $(-1)^{(\ell-1) / 2}(\bmod \ell)$ for $2<\ell \leq p$; then every prime $q \equiv a(\bmod N)$ satisfies (i) and (ii), and we obtain the first statement of the theorem. The second statement follows from the bound $N \leq \exp (p+o(p))$ and Linnik's theorem.

Since +1 is always a quadratic residue modulo $q$, the method of Theorem 2.5 cannot be used to prove the analogous statement for the set $\mathcal{G}(\mathbb{N})$. However, numerical evidence suggests that such a statement is likely to be true.

Question 2.6. Does an analogue of Theorem 2.5 hold if the value set $\mathcal{F}(\mathbb{N})$ is replaced by $\mathcal{G}(\mathbb{N})$ ?

It follows from the classical results of $\mathrm{H}$. Hasse that the set of primes which divide some element of the sequence $\left\{2^{k}+1: k=1,2,3, \ldots\right\}$ has relative asymptotic density $2 / 3$ in the set of all prime numbers (see [2] for an exhaustive survey of results of this kind). This immediately implies that

$$
\#\{\text { primes } q \leq x: 2 / q \notin \mathcal{F}(\mathbb{N})\} \geq(1 / 3+o(1)) \pi(x) .
$$

A slight modification of this argument also works for $\mathcal{G}(\mathbb{N})$ and in fact using some results of [11] one can show that

$$
\#\{\text { primes } q \leq x: 2 / q \notin \mathcal{G}(\mathbb{N})\}=(1+o(1)) \pi(x) .
$$

Question 2.7. Is it true that the lower bound

$$
\#\{\text { prime pairs }(p, q) \text { with } p<q \leq x: p / q \notin \mathcal{F}(\mathbb{N})\} \geq x^{1+\delta}
$$

holds for some absolute constant $\delta>0$ and all sufficiently large values of $x$ ?

\section{Distribution of values}

Theorem 3.1. If $F=\mathcal{F}$ or $F=\mathcal{G}$, then for any $\varepsilon>0$ the following estimate holds:

$$
\#\left\{n \leq x: F(n) \leq x^{1 / u}\right\} \ll \frac{x \log _{2} x}{\log x \log _{3} x}+x \exp (-(1-\varepsilon) u \log u),
$$

where the implied constant in the «-symbol depends only on $\varepsilon$. 
Proof. For a fixed integer $a \neq 0$, let

$$
F_{a}(n)=\frac{P^{+}(n)}{P^{-}(n+a)} \quad(n \geq 1-a)
$$

Since $\mathcal{F}(n)=F_{1}(n)$ and $\mathcal{G}(n)=F_{-1}(n+1)$, it suffices to prove the stated inequality for the function $F=F_{a}$. Let us fix a sufficiently small $\delta>0$. Put

$$
y=x^{1 / u}, \quad v=\min \left\{\frac{u}{1+\delta}, \frac{2 \log _{2} x}{\log _{3} x}\right\}, \quad \text { and } \quad z=x^{1 / v},
$$

and note that $z \geq y^{(1+\delta)}$. Clearly, if $F_{a}(n) \leq y$, then $P^{-}(n+a) \geq P^{+}(n) / y$; hence, either $P^{+}(n) \leq z$ or $P^{-}(n+a)>z / y$. For integers of the first type, we use the bound (see, for example, [14, chapter III.5]):

$$
\Psi(x, z) \leq x \exp (-(1+o(1)) v \log v),
$$

where

$$
\Psi(x, z)=\#\left\{n \leq x: P^{+}(n) \leq z\right\},
$$

and for integers of the second type, we use the bound (see [14, Chapter III.6]):

$$
\Phi(x+a, z / y) \ll \Phi(x, z / y) \ll \frac{x}{\log (z / y)} \leq \frac{x v}{\delta \log x},
$$

where

$$
\Phi(x, z / y)=\#\left\{n \leq x: P^{-}(n)>z / y\right\} .
$$

Taking a sufficiently small $\delta$, after simple calculations, we obtain the stated result.

Theorem 3.2. For a positive real number $x$, the lower bound

$$
\#(\{\mathcal{F}(m): m \leq x\} \cap\{\mathcal{G}(n): n \leq x\}) \gg \frac{x}{\log x}
$$

holds.

Proof. This is clear since all fractions of the form $p / 2=\mathcal{F}(p)=\mathcal{G}(p-1)$ with $2<p \leq x$ are distinct.

\section{Extreme values}

Theorem 4.1. As $x \rightarrow \infty$, each of the inequalities

$$
\mathcal{F}(n) \geq n^{7 / 10}, \quad \mathcal{F}(n) \leq n^{-7 / 10}, \quad \mathcal{G}(n) \geq n^{7 / 10}, \quad \text { and } \quad \mathcal{G}(n) \leq n^{-7 / 10}
$$

holds for $x^{1+o(1)}$ positive integers $n \leq x$. 
Proof. By a well-known result of R. C. Baker and G. Harman [1], for any fixed integer $a \neq 0$, there exists a constant $C>0$ such that the cardinality of the set

$$
\mathcal{P}_{a}(x)=\left\{\text { primes } p \leq x: P^{+}(p-a) \leq p^{0.2961}\right\}
$$

is bounded below by

$$
\# \mathcal{P}_{a}(x)>\frac{x}{(\log x)^{C}}=x^{1+o(1)}
$$

for all sufficiently large values of $x$. In particular, we have

$$
\mathcal{F}(p-1)=\frac{P^{+}(p-1)}{p} \leq p^{-0.7039} \quad \text { and } \quad \mathcal{G}(p-1)=\frac{p}{P^{-}(p-1)} \geq p^{0.7039}
$$

for all $p \in \mathcal{P}_{1}(x)$, and

$$
\mathcal{F}(p)=\frac{p}{P^{+}(p+1)} \geq p^{0.7039} \quad \text { and } \quad \mathcal{G}(p)=\frac{P^{+}(p+1)}{p} \leq p^{-0.7039}
$$

for all $p \in \mathcal{P}_{-1}(x)$. The result follows.

Remark 4.2. Assuming the Elliott-Halberstam conjecture, it is clear that the constant $7 / 10$ can be replaced by $1-\varepsilon$ for any fixed $\varepsilon>0$.

Remark 4.3. We note that $\mathcal{F}(n) \geq 2 /(n+1)$ holds for all $n \geq 2$, and $\mathcal{F}(n)=2 /(n+1)$ if and only if $n+1$ is a Fermat prime. Similarly, $\mathcal{G}(n) \geq 2 / n$ holds for all $n \geq 2$, and $\mathcal{G}(n)=2 / n$ if and only if $n$ is a Mersenne prime.

As a complementary result to Theorem 4.1, we now state the following corollary to Theorem 2.1, which concerns integers $n$ for which $\mathcal{F}(n)$ or $\mathcal{G}(n)$ is close to 1 .

Corollary 4.4. Both of the inequalities

$$
|\mathcal{F}(n)-1| \leq(1+o(1)) \frac{\log _{2} n}{\log n} \quad \text { and } \quad|\mathcal{G}(n)-1| \leq(1+o(1)) \frac{\log _{2} n}{\log n}
$$

hold for infinitely many $n \in \mathbb{N}$.

Proof. By the Prime Number Theorem, there are infinitely many consecutive primes $q<p$ such that

$$
|p-q| \leq(1+o(1)) \log q .
$$

By Theorem 2.1, one can find $m, n \in \mathbb{N}$ with $\max \{m, n\} \leq \exp (p+o(p))$ such that

$$
\mathcal{F}(m)=\mathcal{G}(n)=\frac{p}{q}=1+O\left(\frac{\log q}{q}\right)=1+O\left(\frac{\log p}{p}\right) .
$$

Since $p \geq(1+o(1)) \max \{\log m, \log n\}$, the result follows. 
Remark 4.5. By the recent breakthrough result of D. A. Goldston, J. Pintz, and C. Y. Yıldırım [7], there are infinitely many consecutive primes $q<p$ for which

$$
p=q+O\left(\frac{\log q \log _{4} q}{\log _{2} q}\right)
$$

and this result leads to an obvious improvement in the bound of Corollary 4.4.

Remark 4.6. We observe that

$$
|\mathcal{F}(n)-1| \geq(n+1)^{-1 / 2} \quad(n \geq 3) .
$$

Indeed, if $n+1$ is prime, then $P^{+}(n) \leq n / 2$ and $P^{-}(n+1)=n+1$. Hence, $\mathcal{F}(n)<1 / 2$, and therefore $|\mathcal{F}(n)-1|>1 / 2 \geq(n+1)^{-1 / 2}$ (since $\left.n+1 \geq 4\right)$. On the other hand, if $n+1$ is composite, then $P^{-}(n+1) \leq(n+1)^{-1 / 2}$, and the bound (2) follows from the obvious inequality $|\mathcal{F}(n)-1| \geq 1 / P^{-}(n+1)$.

We believe that for every $\varepsilon>0$ there exists $n$ such that

$$
|\mathcal{F}(n)-1| \leq n^{-1 / 2+\varepsilon}
$$

but we do not know how to attack this problem. Perhaps it follows from standard conjectures about the distribution of prime numbers, such as the Elliott-Halberstam conjecture, but our efforts to find such an argument have not been successful.

\section{References}

[1] R. C. Baker and G. Harman, Shifted primes without large prime factors, Acta Arith. 83 (1998), no. 4, 331-361.

[2] C. Ballot, Density of prime divisors of linear recurrences, Mem. Amer. Math. Soc. 115 (1995), no. 551 .

[3] A. Balog, On triplets with descending largest prime factors, Studia Sci. Math. Hungar. 38 (2001), 45-50.

[4] A. Balog and I. Z. Ruzsa, On an additive property of stable sets, Sieve Methods, Exponential Sums, and Their Applications in Number Theory (Cardiff, 1995), London Math. Soc. Lecture Note Ser., vol. 237, Cambridge Univ. Press, Cambridge, 1997, pp. 55-63.

[5] C. Dartyge, G. Martin, and G. Tenenbaum, Polynomial values free of large prime factors, Period. Math. Hungar. 43 (2001), no. 1-2, 111-119.

[6] P. Erdős and C. Pomerance, On the largest prime factors of $n$ and $n+1$, Aequationes Math. 17 (1978), no. 2-3, 311-321.

[7] D. A. Goldston, J. Pintz, and C. Y. Yıldırım, Small gaps between primes, II (2005), preprint.

[8] H. Halberstam and H.-E. Richert, Sieve methods, London Mathematical Society Monographs, vol. 4, Academic Press, London-New York, 1974.

[9] J. Haristoy, Équations diophantiennes exponentielles, Prépublication de l'Institut de Recherche Mathématique Avancée, vol. 2003/29, Université Louis Pasteur, Département de Mathématique, Institut de Recherche Mathématique Avancée, Strasbourg, 2003. Thèse, University of Strasbourg I (Louis Pasteur), Strasbourg, 2003. 
[10] A. Hildebrand, On integer sets containing strings of consecutive integers, Mathematika 36 (1989), no. 1, 60-70.

[11] F. Pappalardi, Square free values of the order function, New York J. Math. 9 (2003), 331-344.

[12] J. B. Rosser and L. Schoenfeld, Approximate formulas for some functions of prime numbers, Illinois J. Math. 6 (1962), 64-94.

[13] C. Siegel, Approximation algebraischer Zahlen, Math. Z. 10 (1921), no. 3-4, 173-213.

[14] G. Tenenbaum, Introduction to analytic and probabilistic number theory, Cambridge Studies in Advanced Mathematics, vol. 46, Cambridge University Press, Cambridge, 1995.

[15] N. M. Timofeev, Polynomials with small prime divisors, Taškent. Gos. Univ. Naučn. Trudy (1977), no. 548 Voprosy Mat., 87-91, 145 (Russian). 